\title{
Novel membrane cell projection defects in Wiskott-Aldrich syndrome B cells
}

\author{
NURIA ANDREU ${ }^{1}$, JOSEP M. ARAN ${ }^{2}$ and CRISTINA FILLAT ${ }^{1}$ \\ ${ }^{1}$ Programa Gens i Malaltia, Centre de Regulació Genòmica-CRG-UPF, Parc de Recerca Biomèdica (PRBB), Barcelona; \\ ${ }^{2}$ Centre de Genètica Mèdica i Molecular-IDIBELL, L'Hospitalet de Llobregat, Barcelona, Spain
}

Received June 1, 2007; Accepted July 13, 2007

\begin{abstract}
Wiskott-Aldrich syndrome (WAS) is an X-linked immunodeficiency characterized by microthrombocytopenia, eczema, recurrent infections, autoimmune disorders and an increased incidence of malignancies. This complex phenotype results from mutations in the WASP gene. WASP is a key member of a protein family that links signaling pathways to actin cytoskeleton reorganization by activating Arp2/3mediated actin polymerization. Actin polymerization defects have been extensively defined in WAS T cells and also in dendritic cells and macrophages, but few reports have concentrated on WAS B cells. In the present study, we investigated cytoskeleton abnormalities in WAS B cell lines. For the first time we report alterations in the capacity of these cells to extend filopodia in response to bradykinin stimuli and an impairment in the formation of long pseudopodia under basal conditions. Such alterations most probably result from a WASP dysfunction, given that a retroviral gene transfer of a corrected form of the WASP gene was able to rescue the abnormal phenotypes.
\end{abstract}

\section{Introduction}

The Wiskott-Aldrich syndrome protein (WASP) is the 502amino acid product of the gene defective in the X-linked inherited immunodeficiency known as Wiskott-Aldrich syndrome (WAS, MIM no. 301000) (1). WASP is predominantly a cytoplasmic protein, expressed only in hematopoietic cells. The majority of WASP gene mutations

Correspondence to: Dr Cristina Fillat, Centre de Regulació Genòmica, Dr Aiguader 88, 08003 Barcelona, Spain

E-mail: cristina.fillat@crg.es

Abbreviations: WAS, Wiskott-Aldrich syndrome; WASP, Wiskott-Aldrich syndrome protein; XLT, X-linked thrombocytopenia; B-LCLs, Epstein-Barr virus (EBV)-transformed B lymphoblastoid cell lines

Key words: Wiskott-Aldrich syndrome protein, actin cytoskeleton, pseudopodia, retrovirus result in a loss of protein function and give rise to a spectrum of clinical phenotypes determined by the severity or location of the molecular defect, and also probably by secondary genetic and environmental factors (2).

The biological mechanisms responsible for the pathophysiology of WAS have been shown to be linked to the regulation of actin polymerization in hematopoietic cells (3). WASP protein is known to be a member of a unique family sharing similar domain structures, responsible for the transduction of signals from the cell membrane to the actin cytoskeleton. The interaction between WASP, the Rho family GTPase Cdc42, and the cytoskeletal organizing complex Arp2/3, is probably critical to many of these functions, which when disturbed, translate into measurable defects of cell signaling, polarization, motility, and phagocytosis (3).

A variety of actin-based cell surface membrane projections has been described in hematopoietic cells, including microvilli, lamellipodia, microspikes and filopodia (4). The Rho family of GTPases and the WASP family have been shown to be involved in the signal-transduction pathways that induce the formation of these projections. For instance, N-WASP and WASP mediate Cdc42-induced filopodia formation (5), and WAVE/Scar (WASP family verprolin-homologous protein) mediates membrane ruffling through Rac activation (6).

In addition to their classical projections, it has also been reported that hematopoietic cells can display a variety of dynamic pseudopods of diverse morphology $(7,8)$. Tenupodia are very thin, adhere to the plate surface, and can bifurcate multiple times. Magnupodia, also known as cytonemes or proteopodia, are large, slightly thicker than tenupodia, and do not adhere to the surface on which the cells are cultured. Both types of podia can exceed $300 \mu \mathrm{m}$ in length. Such structures are believed to participate in cell navigation and cell-cell communication $(7,8)$.

The participation of WASP in actin polymerization is well known, and the alteration thereof in WAS hematopoietic cells, such as T cells, dendritic cells and neutrophils, has been studied extensively. However, little is known of the consequences of WASP defects on the membrane projections of B cells. In the present study, we examined actin-based cytoskeleton defects in B cells from WAS patients affecting cell membrane morphology and the feasibility of rescue by retrovirus-mediated WASP gene transfer. 


\section{Materials and methods}

Cells and cell lines. The murine cell line NIH-3T3 and the murine packaging cell line PA317 were maintained in DMEM (Invitrogen, Paisley, UK), supplemented with 10\% FBS, penicillin $(100 \mathrm{U} / \mathrm{ml})$, streptomycin $(100 \mu \mathrm{g} / \mathrm{ml})$ and glutamine (2 mM) (Invitrogen).

Blood samples from WAS patients and control subjects were obtained following standard ethical procedures. Epstein-Barr virus (EBV)-transformed B lymphoblastoid cell lines (B-LCLs) were generated as described previously (9). The cells lines were maintained in RPMI-1640 medium (Invitrogen), supplemented with 10\% heat-inactivated FBS, penicillin $(100 \mathrm{U} / \mathrm{ml})$ and streptomycin $(100 \mu \mathrm{g} / \mathrm{ml})$ (Invitrogen).

The WASP gene mutations of the five patients studied have been previously described (10). The mutations were as follows: patient E2, $291 \mathrm{G}>\mathrm{A}$, resulting in $\mathrm{R} 86 \mathrm{H}$; patient $\mathrm{E} 2 \mathrm{~V}, 257 \mathrm{G}>\mathrm{A}$, resulting in V75M; patient E4, 431 G>A, resulting in $\mathrm{E} 133 \mathrm{~K}$; patient $\mathrm{E} 10,995 \mathrm{C}>\mathrm{T}$, resulting in R321X; and patient E11, $1487 \mathrm{G}>\mathrm{A}$, resulting in D458N.

Retroviral vectors and viral production. The retroviral vectors used in this study were derived from the Moloney Murine Leukaemia Virus (MMLV) and contained the neomycin phosphotransferase gene (neo) as a selectable marker.

The pLHWSN retroviral vector was constructed by subcloning the $H A-W A S P$ cDNA in the pLXSN plasmid (Clontech, Palo Alto, CA). In brief, the WASP cDNA was engineered to introduce: i) an XhoI restriction site followed by an NcoI site containing the ATG translation initiation codon from WASP on its 5'-end, and ii) a BspMI-SphI-BamHI adaptor on its 3'-end, introduced into the XhoI/BamHI pBluescript (Stratagene, La Jolla, CA) to generate pWASP. Next, the pWASP was digested with blunt-ended NcoI/XbaI. The corresponding WASP fragment was then ligated to a blunt-ended BamHI/XbaI pHA1 [donated by S. de la Luna, (11)] expression vector. In this step a double HA epitope tag was placed in frame with the 5'-end of the WASP cDNA to generate pHA-WASP. Finally, the $H A-W A S P$ cDNA was excised from the pHA-WASP plasmid as a blunt-ended HindIII/BamHI fragment and ligated into blunt-ended EcoRI/BamHI sites from the pLXSN vector. The resultant plasmid was termed pLHWSN.

For viral production, retroviral vectors were transfected using the calcium/phosphate-DNA precipitation method into the amphotropic packaging cell line PA317, plated in $100 \mathrm{~mm}^{2}$ cell culture dishes (Corning, Schiphol-Rijk, The Netherlands) and subjected to $\mathrm{G} 418$ (Invitrogen) selection $(800 \mu \mathrm{g} / \mathrm{ml})$ for 14 days. G418-resistant clones were isolated by limiting dilution, and viral production was determined by dot blot as described by Onodera et al (12). The clones with high intensity signals were tested for their viral titer on NIH-3T3. The viral producer clone with the highest viral titer $\left[6 \times 10^{5}\right.$ colonyforming unit (c.f.u) per ml] was used in subsequent studies.

Likewise, we generated control retrovirus producer cells with the empty pLXSN vector which carried only the neo gene.

Transduction of EBV cell lines. EBV-transformed B-LCLs from the WAS patients $\left(4 \times 10^{6}\right.$ cells/sample) were exposed to
$3 \mathrm{ml}$ of virus-containing medium (pLHWSN or pLXSN) in the presence of $8 \mu \mathrm{g} / \mathrm{ml}$ polybrene. The cells were transduced once a day for 3 consecutive days, following a spin-infection procedure (13). Five days after the final exposure to the viral supernatant, the cells were selected in G418 (800 $\mu \mathrm{g} / \mathrm{ml})$ for 10 days. The resistant cell pools were harvested and used for subsequent experiments.

Western blot analysis. The cells were washed twice in phosphate-buffered saline (PBS), lysed in $100 \mu 1$ of cold lysis buffer $(50 \mathrm{mM}$ Tris- $\mathrm{HCl}, \mathrm{pH} 7.5 ; 1 \%$ Nonidet P-40; 0.25\% sodium deoxycholate; and 1 tablet of Complete ${ }^{\mathrm{TM}}$ protease inhibitor (Roche Diagnostics, Mannheim, Germany) applying 3 freeze-thaw cycles. The resulting lysate was centrifuged at $12,000 \times \mathrm{g}$ for $5 \mathrm{~min}$, and the protein concentration was determined from the cleared lysate (BCA assay; Pierce, Rockford, IL). Before loading onto the gel, the appropriate amount of cell lysate was mixed with an equal amount of loading buffer and boiled at $100^{\circ} \mathrm{C}$ for $10 \mathrm{~min}$. Total protein $(50 \mu \mathrm{g})$ from each sample was loaded on a $10 \%$ SDSpolyacrylamide gel and transferred onto Hybond ${ }^{\mathrm{TM}}-\mathrm{C}$ extrasupported nitrocellulose membranes (Amersham Biosciences, Freiburg, Germany) using a Bio-Rad gel transfer apparatus (Bio-Rad Laboratories, Barcelona, Spain). The membranes were blocked in 5\% non-fat dried milk dissolved in TBS-T (10 mM Tris pH 7.5, $100 \mathrm{mM} \mathrm{NaCl}, 0.1 \%$ Tween-20) for $1 \mathrm{~h}$ at room temperature and then incubated overnight at $4^{\circ} \mathrm{C}$ with the primary antibody, a mouse monoclonal anti-HA (Berkeley Antibody Co., Berkeley, CA), diluted 1:1000 in $5 \%$ powdered milk in TBS-T. The secondary antibody, a rabbit anti-mouse immunoglobulin $G$ conjugated with horseradish peroxidase (Dako, Glostrup, Denmark) was applied at a 1:1000 dilution in 5\% powdered milk in TBS-T at room temperature for $1 \mathrm{~h}$ after removal of the primary antibody. The HA-WASP protein $(70 \mathrm{kDa})$ was visualized by enhanced chemiluminescence (Pierce).

Immunocytochemistry. The cells were fixed in 4\% paraformaldehyde in PBS at room temperature for $20 \mathrm{~min}$, washed with PBS, and permeabilized with $0.1 \%$ Triton X-100 in PBS at room temperature for $10 \mathrm{~min}$. After blocking with $2 \%$ BSA (Sigma, St. Louis, MO) in PBS, the cells were doublelabeled for WASP and F-actin. For WASP staining, the cells were incubated with mouse anti-HA antibody (dilution 1:1000) (Berkeley Antibody Co.), washed with PBS, and incubated with mouse anti-IgG FITC-conjugated antibody (dilution 1:200) (Southern Biotechnologies Associates, Birmingham, AL). F-actin labeling was carried out sequentially, incubating with $1 \mu \mathrm{g} / \mathrm{ml}$ TRITC-conjugated phalloidin (Sigma) after washing off the secondary antibody. Finally, the cells were washed with PBS, mounted in Vectashield (Vector Laboratories, Burlingame, CA) and visualized under a fluorescent microscope. The images were captured with an inverted Leica TCS SP2 confocal scanning laser microscope.

\section{Analysis of the actin cytoskeleton from EBV-transformed B-LCLs.}

Bradykinin stimulation. Cultured lymphocytes were serum-starved for $24 \mathrm{~h}$, followed by stimulation with $1 \mu \mathrm{g} / \mathrm{ml}$ 
bradykinin (Calbiochem, Merck, Darmstadt, Germany) for $15 \mathrm{~min}$ at $37^{\circ} \mathrm{C}$ in $5 \% \mathrm{CO}_{2}$. The cells were then washed and used to prepare cytospin slides. Immunocytochemistry was performed to detect F-actin, using TRITC-conjugated phalloidin (Sigma).

Cell membrane labeling with PKH2. The PKH2 membrane dye (Sigma) was used to visualize the pseudopodia. One million cells from each cell line were washed in PBS twice to remove all traces of serum and re-suspended in $1 \mathrm{ml}$ of diluent A (supplied with the dye kit). PKH2 membrane dye $(6 \mu 1)$ (1-mM stock solution) was diluted in another $1 \mathrm{ml}$ of diluent A before mixing with the cells. The final cell concentration was $5 \times 10^{5} / \mathrm{ml}$, and the labeling was terminated after $6 \mathrm{~min}$ by adding $2 \mathrm{ml}$ heat-inactivated FBS. After cessation of the reaction, the cells were washed in PBS and re-suspended in their original media. Aliquots $(100 \mu 1)$ of cell suspension were placed onto poly-L-lysine-coated slides and allowed to dry. Drying was performed to preserve the fragile podia structures since conventional fixing protocol results in damaged and missing podia (7). The cells were imaged under a fluorescent microscope (Leica) with x40 and x63 objectives. Digitized images were captured with a cooled CCD Camera (Leica). The frequency of cells forming podia was determined by counting a minimum of 100 cells.

Image processing. Podia were clearly visible with the fluorescent membrane dye $\mathrm{PKH} 2$ under high magnification. The fluorescent podia were very dim compared to the bright cells from which they emanated. Thus, the contrast of the fluorescent images was enhanced to make the podia more clearly visible. With the enhanced contrast, the quality of the image of the cells was poorer, and small pieces of membrane fragments and debris created during the staining process appeared as bright objects.

\section{Results}

Characterization of EBV-WAS cells transduced with WASP retrovirus. Epstein-Barr virus (EBV)-transformed B lymphoblastoid cell lines (B-LCLs) from WAS patients E2, E2V, E4 and E11 were transduced with recombinant WASP (pLHWSN) or control (pLXSN) retroviral vectors. WASP protein was expressed in WASP-transduced cells and colocalized to F-actin filaments (Fig. 1).

Actin microspike formation after bradykinin stimulation is defective in B cells from WAS patients and is rescued by pLHWSN retroviral transduction. The bioactive peptide bradykinin induces the formation of actin-containing microspikes or filopodia at the cell periphery (14). This process has been studied in non-hematopoietic cells and was shown to be dependent on Cdc42, a GTPase that binds to hematopoietic-cell-specific WASP $(15,16)$, causing a conformational change that allows WASP to interact with the Arp2/3 complex and initiate actin polymerization (17-19).

To investigate the involvement of WASP in the formation of filopodia in B cells, we stimulated serum-starved B cell lines with bradykinin and monitored the changes in their actincontaining structures, staining with rhodamine-conjugated phalloidin. Control B cells formed microspikes or filopodia in response to bradykinin stimulation. However, B cells
A
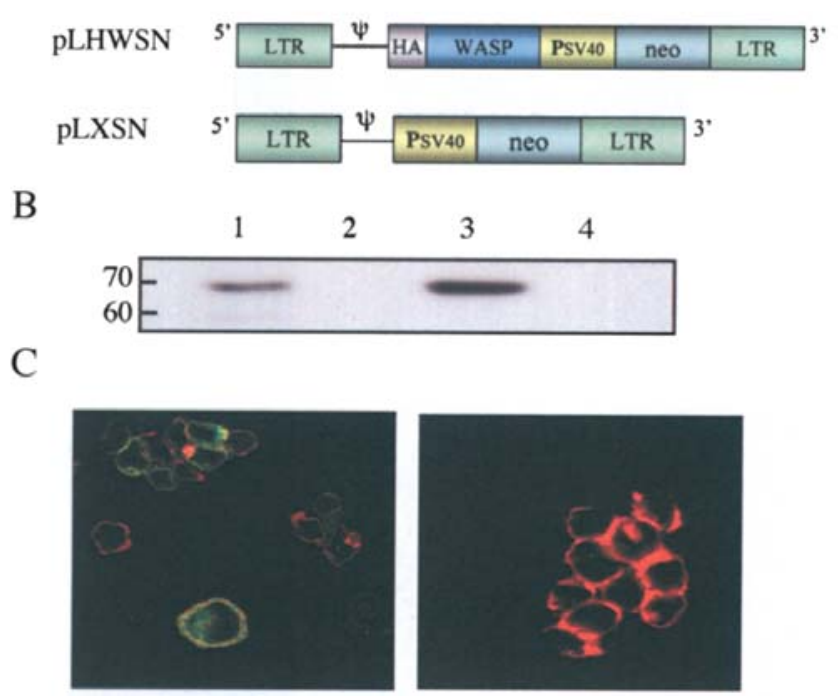

Figure 1. HA-WASP expression in B cells from WAS patients transduced with the pLHWSN recombinant retrovirus. (A) Schematic representation of retroviral vectors. pLHWSN: encoding the HA-tagged WASP under the control of the MMLV LTR and the neomycin resistance gene under the control of the SV40 internal promoter. pLXSN: control retrovirus encoding only for the neomycin resistance gene. (B) Western blot of cell lysates from lymphoblastoid cell lines carrying WASP mutations E2 (R86H) (lanes 1 and 2) and E4 (E133K) (lanes 3 and 4), transduced with pLHWSN (lane 1, E2W and 3, E4W), or transduced with the control retrovirus pLXSN (lane 2, E2n and 4, E4n). HA-WASP (70 KDa) was detected with a mouse monoclonal anti-HA antibody. (C) Confocal microscopy. Immunocytochemistry of the lymphoblastoid cell line E4 transduced with pLHWSN retrovirus $(\mathrm{E} 4 \mathrm{~W})$ and $\mathrm{pLXSN}$ control retrovirus $(\mathrm{E} 4 \mathrm{n})$. Cells were stained with phalloidin-TRITC (red) to detect F-actin and incubated with anti-HA antibody plus anti-IgG FITC-conjugated antibody to detect HA-WASP (green). Original magnification, x1000

derived from WAS patients did not form microspikes (Fig. 2A). Notably, whereas WAS B cell lines transduced with the WASP retrovirus (pLHWSN) recovered the capacity to form actin microspikes (or filopodia) after bradykinin stimulation, no filopodia formation was observed in WAS B cells transduced with the control retrovirus (pLXSN) (Fig. 2B). These results suggest that WASP may play an important role in the formation of bradykinin-induced filopodia.

Pseudopodia formation is defective in WAS B cells and is rescued by WASP retroviral gene transfer. Pseudopodia have been identified in several hematopoietic cells as membrane extensions that are extremely fragile and sensitive to strong fixation. They are commonly visualized by staining with a fluorescent membrane dye. We evaluated the ability of B cells from WAS patients to form pseudopodia after $\mathrm{PKH} 2$ staining. Five B cell lines derived from healthy control subjects and five B cell lines derived from WAS patients (E2, E2V, E4, E10 and E11) were used in the study. Approximately $10 \%$ of control cells from each individual had the capacity to form short and long podia (pseudopodia) in basal conditions. A single cell was found capable of extending either single or multiple protrusions, and along the threads bulbs were sometimes observed. These podia were dynamic and occasionally connected different cells. However, none of the 
A
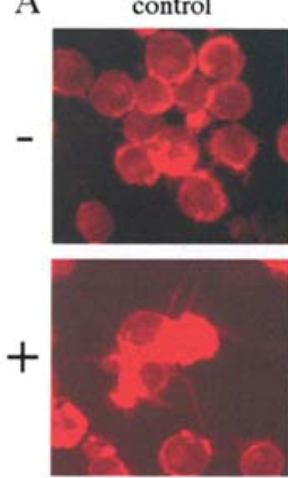

B

E2n
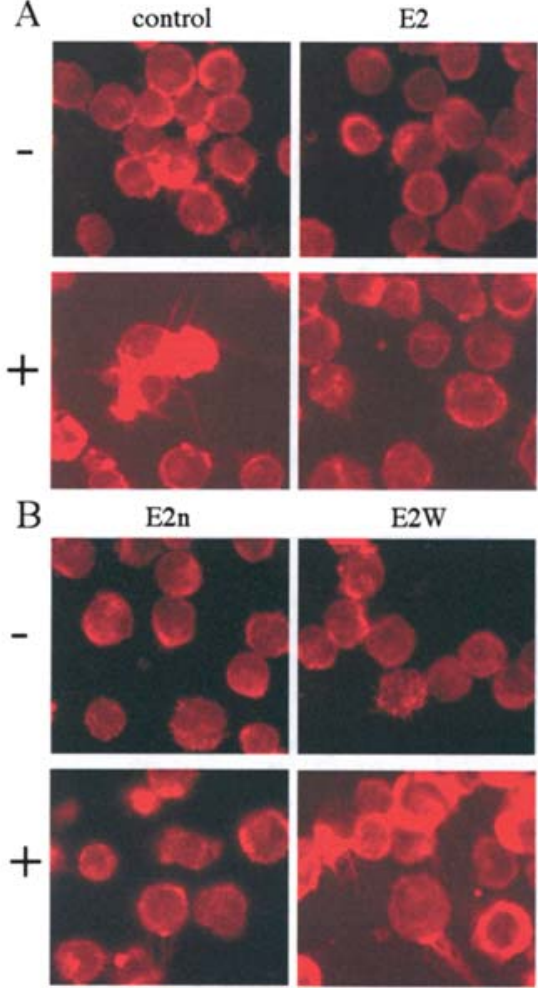

E2W
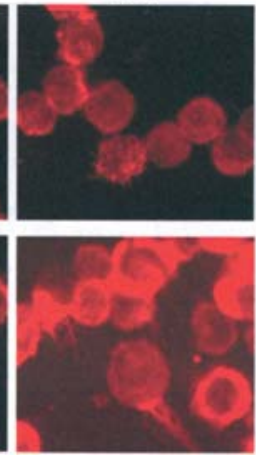

Figure 2. WASP induces the formation of filopodium after bradykinin stimulation. (A) F-actin distribution in control and WAS B cell lines (E2, E4), either unstimulated (-), or stimulated (+) for $15 \mathrm{~min}$ with $1 \mu \mathrm{g} / \mathrm{ml}$ bradykinin. Original magnification, x600. (B) F-actin distribution in unstimulated transduced WAS B-LCLs (-) and in transduced WAS B-LCLs, stimulated with bradykinin (+). Images of WAS B cell lines transduced with control retrovirus pLXSN (E2n) or with WASP retroviral vector pLHWSN (E2W and E4W).

5 B cell lines derived from WAS patients were able to form these membrane projections (Fig. 3A and C). Notably, B cell lines from WAS patients, transduced with WASP-retroviral vector also displayed thin long podia, morphologically similar to those seen in the control cells with the same basic characteristics. In basal conditions, WAS B cells expressing the recombinant WASP protein were capable of attaining a percentage of pseudopodia similar to that of normal B cells. Such structures were not rescued in WAS B cells transduced with the control retrovirus pLXSN (Fig. 3B and C).

All the above results indicated that WAS B cells do present remarkable actin cytoskeleton defects, but, more importantly, we demonstrated that they are rescued by retroviral vectormediated WASP gene transfer.

\section{Discussion}

Earlier investigations of WASP-deficient cells have led to the recognition of a variety of morphological and functional abnormalities in several hematopoietic cell lineages. The abnormalities reported in these cells are often associated with defects in the rearrangement of the actin cytoskeleton in response to extracellular signals (20).

Lymphocytes from WAS patients are morphologically abnormal. They have reduced numbers of surface microvilli, show variably impaired transduction of early activation
A

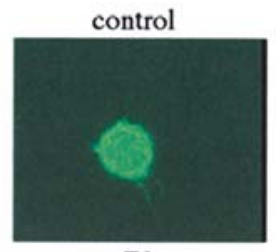

E2

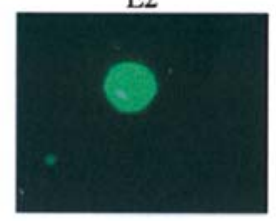

B

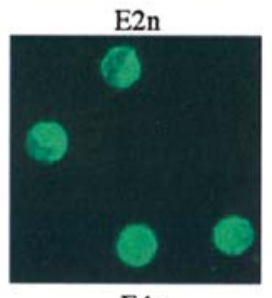

EAn

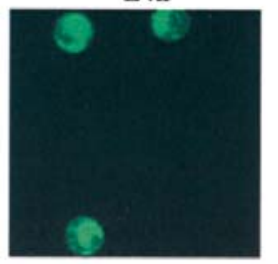

C

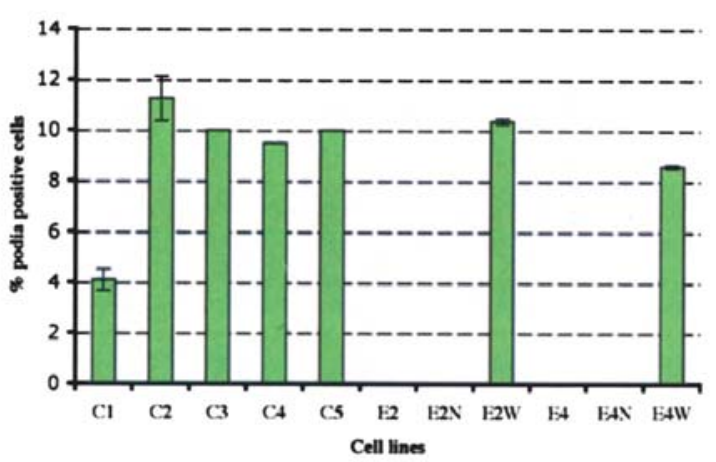

Figure 3. Pseudopodia extensions in EBV-transformed B cell lines. (A) Representative images showing PKH2 fluorescent staining of B-EBV cells. Photographs of control or WAS-derived cell lines (E2 and E4). (B) B cell lines from WAS patients, E2 and E4, transduced with control retrovirus (E2n and E4n) or with WASP retroviral vector (E2W and E4W). (C) Quantification of lymphocytes of normal individuals and WAS patients which presented pseudopodia after PKH2 staining. C1, C2, C3, C4 and C5 are control B cell lines.

signals through antigen receptors on both $\mathrm{B}$ and $\mathrm{T}$ cells, and have abnormal actin and TCR capping (20). The few existing studies on WAS B lymphoblastoid cell lines have shown abnormal cytoarchitecture, reduced proliferative responses, and disturbances in early signaling events (21-23). In the present study we investigated cytoskeleton alterations in WASP-deficient B cells, in particular the formation of filopodia and pseudopodia and the feasibility of rescuing such abnormalities with WASP retroviral gene transfer.

EBV-transformed B cells from normal individuals showed the formation of filopodia after bradykinin stimulation. However, WASP-deficient B lymphocytes E2 and E4 were 
impaired by bradykinin-dependent induction. Evidence suggests that the formation of filopodia is rescued in E2 and E4 cell lines after retroviral transduction with a wild-type WASP gene. It has been proposed that bradykinin acts by activating $\mathrm{Cdc} 42$ which, in turn, stimulates WASP/N-WASP activity and induces actin polymerization to generate filopodia $(5,14,19,24)$. While the role of $\mathrm{N}$-WASP in this signaling cascade seems to be clear, the participation of WASP has been questioned. Miki et al showed that after coexpression of N-WASP and Cdc42, extremely long microspikes were formed. Whereas, in a similar experiment, after coexpression of WASP and $\mathrm{Cdc} 42$, the formation of microspikes was repressed. From this data the authors concluded that WASP is probably not a downstream effector of filopodium formation (5). This data seems to be in contrast with our findings, but as these experiments were performed with COS-7 cells, the existence of a cell-selective-mediated response is speculated. In fact, the impairment of WASPdeficient B lymphocytes in filopodia formation was also observed in response to IL-4 and CD40 stimuli (25), thus suggesting that, at least in B cells, WASP may play a role in the induction of filopodia.

WASP has also been implicated in the formation of other membrane protrusions in hematopoietic cells, such as podosomes in macrophages $(26,27)$ and microvilli in T cells (28). In the present study we showed that WASP was also involved in the formation of pseudopodia. We observed that, whereas EBV-transformed control B cells were active in the formation of short and long pseudopodia, such function was impaired in WASP-deficient B cells. However, by the expression of exogenous wild-type WASP in these cells, the ability to form pseudopodia was restored.

Little is known about the physiological function of pseudopodia, although a role in navigation and migration, in long distance cell-cell interaction, or even in controlled cell death processes of hematopoietic cells has been proposed (7). Up until now, these long thin podia have only been observed in human stem cells and in certain hematopoietic cell lines. This suggests that the formation of such pseudopodia may be lineage or differentiation stage-specific. Two different morphologies of pseudopodia have been reported (7). Magnupod extensions, described as thin, occurring at large distances $(>300 \mu \mathrm{m})$ from the main cell body and showing rapid dynamic behavior, have been proposed by several groups to be equivalent to the structures known as cytonemes (29) or proteopodia $(4,30)$. However, tenupodia correspond to those previously described as 'retraction fibers' $(30,31)$. Cytonemes have already been described in neutrophils $(29,32)$ and also in B lymphocytes $(33)$. These B cell cytonemes, rich in lipid rafts and actin filaments, are proposed to play a role in long-range communication and/or transportation of signaling molecules during the immune response (33). Collectively, the type of pseudopodia that we describe in the present study, impaired in WAS-deficient B lymphocytes, could in fact be cytonemes.

To summarize, our results underline the involvement of WASP in the formation of bradykinin-stimulated filopodia and pseudopodia in EBV-transformed B cells. Remarkably, retroviral vector-mediated WASP gene transfer rescued these defects in WAS B cells. These data provide further evidence that WAS gene therapy is a feasible approach to treating this life-threatening disorder.

\section{Acknowledgements}

This study was supported by Fundació La Marató de TV3 (grant no. 99/3510), CIBER ref. CB06/07/0089 and by Generalitat de Catalunya (SGR0500008). N.A. was supported by a contract from the Fondo de Investigación Sanitaria. We would like to thank Ana Carreras and Rafael de Cid for providing the control B cell lines.

\section{References}

1. Derry JM, Ochs HD and Francke U: Isolation of a novel gene mutated in Wiskott-Aldrich syndrome. Cell 78: 635-644, 1994.

2. Burns S, Cory GO, Vainchenker W and Thrasher AJ: Mechanisms of WASp-mediated hematologic and immunologic disease. Blood 104: 3454-3462, 2004.

3. Thrasher AJ and Kinnon C: The Wiskott-Aldrich syndrome. Clin Exp Immunol 120: 2-9, 2000.

4. Frimberger AE, McAuliffe CI, Werme KA et al: The fleet feet of haematopoietic stem cells: rapid motility, interaction and proteopodia. Br J Haematol 112: 644-654, 2001.

5. Miki H, Sasaki T, Takai Y and Takenawa T: Induction of filopodium formation by a WASP-related actin-depolymerizing protein N-WASP. Nature 391: 93-96, 1998.

6. Miki H, Suetsugu S and Takenawa T: WAVE, a novel WASPfamily protein involved in actin reorganization induced by Rac. EMBO J 17: 6932-6941, 1998.

7. Francis K, Ramakrishna R, Holloway W and Palsson BO: Two new pseudopod morphologies displayed by the human hematopoietic $\mathrm{KG}$ 1a progenitor cell line and by primary human CD34(+) cells. Blood 92: 3616-3623, 1998.

8. Holloway W, Martinez AR, Oh DJ, Francis K, Ramakrishna R and Palsson BO: Key adhesion molecules are present on long podia extended by hematopoietic cells. Cytometry 37: 171-177, 1999.

9. Neitzel H: A routine method for the establishment of permanent growing lymphoblastoid cell lines. Hum Genet 73: 320-326, 1986.

10. Fillat C, Espanol T, Oset M, Ferrando M, Estivill X and Volpini V: Identification of WASP mutations in 14 Spanish families with Wiskott-Aldrich syndrome. Am J Med Genet 100: 116-121, 2001.

11. de la Luna S, Allen KE, Mason SL and La Thangue NB: Integration of a growth-suppressing BTB/POZ domain protein with the DP component of the E2F transcription factor. EMBO J 18: 212-228, 1999.

12. Onodera M, Yachie A, Nelson DM, Welchlin H, Morgan RA and Blaese RM: A simple and reliable method for screening retroviral producer clones without selectable markers. Hum Gene Ther 8: 1189-1194, 1997.

13. Kotani H, Newton PB III, Zhang S, et al: Improved methods of retroviral vector transduction and production for gene therapy. Hum Gene Ther 5: 19-28, 1994.

14. Kozma R, Ahmed S, Best A and Lim L: The Ras-related protein $\mathrm{Cdc} 42 \mathrm{Hs}$ and bradykinin promote formation of peripheral actin microspikes and filopodia in Swiss 3T3 fibroblasts. Mol Cell Biol 15: 1942-1952, 1995.

15. Kolluri R, Tolias KF, Carpenter CL, Rosen FS and Kirchhausen T: Direct interaction of the Wiskott-Aldrich syndrome protein with the GTPase Cdc42. Proc Natl Acad Sci USA 93: 5615-5618, 1996.

16. Symons M, Derry JM, Karlak B, et al: Wiskott-Aldrich syndrome protein, a novel effector for the GTPase CDC42Hs, is implicated in actin polymerization. Cell 84: 723-734, 1996.

17. Kim AS, Kakalis LT, Abdul-Manan N, Liu GA and Rosen MK: Autoinhibition and activation mechanisms of the WiskottAldrich syndrome protein. Nature 404: 151-158, 2000.

18. Miki H, Miura K and Takenawa T: N-WASP, a novel actindepolymerizing protein, regulates the cortical cytoskeletal rearrangement in a PIP2-dependent manner downstream of tyrosine kinases. EMBO J 15: 5326-5335, 1996. 
19. Rohatgi R, Ho HY and Kirschner MW: Mechanism of N-WASP activation by $\mathrm{CDC} 42$ and phosphatidylinositol 4, 5bisphosphate. J Cell Biol 150: 1299-1310, 2000.

20. Thrasher AJ: WASP in immune-system organization and function. Nat Rev Immunol 2: 635-646, 2002.

21. Simon HU, Mills GB, Hashimoto S and Siminovitch KA: Evidence for defective transmembrane signaling in B cells from patients with Wiskott-Aldrich syndrome. J Clin Invest 90: 1396-1405, 1992.

22. Facchetti F, Blanzuoli L, Vermi W, et al: Defective actin polymerization in EBV-transformed B-cell lines from patients with the Wiskott-Aldrich syndrome. J Pathol 185: 99-107, 1998.

23. Candotti F, Facchetti F, Blanzuoli L, Stewart DM, Nelson DL and Blaese RM: Retrovirus-mediated WASP gene transfer corrects defective actin polymerization in B cell lines from Wiskott-Aldrich syndrome patients carrying 'null' mutations. Gene Ther 6: 1170-1174, 1999.

24. Higgs HN and Pollard TD: Activation by Cdc42 and PIP(2) of Wiskott-Aldrich syndrome protein (WASp) stimulates actin nucleation by Arp2/3 complex. J Cell Biol 150: 1311-1320, 2000.

25. Westerberg L, Greicius G, Snapper SB, Aspenstrom P and Severinson E: Cdc42, Rac1, and the Wiskott-Aldrich syndrome protein are involved in the cytoskeletal regulation of $B$ lymphocytes. Blood 98: 1086-1094, 2001.

26. Linder S, Nelson D, Weiss M and Aepfelbacher M: WiskottAldrich syndrome protein regulates podosomes in primary human macrophages. Proc Natl Acad Sci USA 96: 9648-9653, 1999.
27. Burns S, Thrasher AJ, Blundell MP, Machesky L and Jones GE: Configuration of human dendritic cell cytoskeleton by Rho GTPases, the WAS protein, and differentiation. Blood 98: 1142-1149, 2001.

28. Kenney D, Cairns L, Remold-O'Donnell E, Peterson J, Rosen FS and Parkman R: Morphological abnormalities in the lymphocytes of patients with the Wiskott-Aldrich syndrome. Blood 68: 1329-1332, 1986

29. Galkina SI, Sud'ina GF and Ullrich V: Inhibition of neutrophil spreading during adhesion to fibronectin reveals formation of long tubulovesicular cell extensions (cytonemes). Exp Cell Res 266: 222-228, 2001

30. Francis K, Palsson B, Donahue J, Fong S and Carrier E: Murine Sca-1(+)/Lin(-) cells and human KG1a cells exhibit multiple pseudopod morphologies during migration. Exp Hematol 30: 460-463, 2002.

31. Rabinovitz I and Mercurio AM: The integrin alpha6beta4 functions in carcinoma cell migration on laminin-1 by mediating the formation and stabilization of actin-containing motility structures. J Cell Biol 139: 1873-1884, 1997.

32. Galkina SI, Molotkovsky JG, Ullrich V and Sud'ina GF: Scanning electron microscopy study of neutrophil membrane tubulovesicular extensions (cytonemes) and their role in anchoring, aggregation and phagocytosis. The effect of nitric oxide. Exp Cell Res 304: 620-629, 2005.

33. Gupta $\mathrm{N}$ and DeFranco AL: Visualizing lipid raft dynamics and early signaling events during antigen receptor-mediated Blymphocyte activation. Mol Biol Cell 14: 432-444, 2003. 